ORIGINAL ARTICLE

\title{
The Association between Morning Blood Pressure Surge and Microalbuminuria in Normotensive Type 2 Diabetic Patients
}

\author{
Ragab Abdel-Salam Mahfouz, Islam El-Sayyed Mohammed Shehata', Ismail Mohammed \\ Ibrahim ', Abdel-Rahman Osama Abdel-Kareem ${ }^{\mathrm{I}} *$

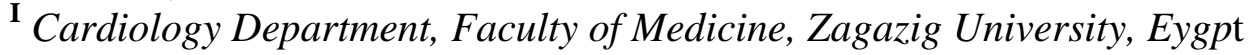

\begin{abstract}
*Corresponding Author;
Abdel-Rahman Osama

Abdel-Kareem

Cardiology Department, Faculty of Medicine, Zagazig University, Egypt ao.khattaby.sci@gmail.com
\end{abstract}

Submit Date 2019-07-15

Revise Date 2019-07-30

Accept Date 2019-08-03

ABSTRACT
Background: The slight fluctuations in blood pressure (BP) regulation even
without advancing to abnormal values appear to be related to more
accelerated cardiovascular complications in patients with type 2 diabetes
mellitus (T2DM). Morning blood pressure surge (MBPS) has been
increasingly reported to provide a significant predictor of cardiovascular
disease (CVD) risk. We aim to study the whether MBPS is associated with
increased levels of microalbuminuria in normotensive T2DM patients.
Methods: Normotensive T2DM patients were subjected to ambulatory blood
pressure monitoring (ABPM) and urine spot sampling to assess urine
albumin creatinine ratio (UACR). Results: $32 \%$ of patients had
microalbuminuria with a highly significant moderate positive correlation
between UACR with DM duration, MBPS and average 2-hours systolic
blood pressure (SBP) with ( $\mathrm{r}=0.342)$ p-value $<0.01$, $(\mathrm{r}=0.361)$ p-value $<$
0.01 and ( $\mathrm{r}=0.324)$ p-value <0.01 respectively. Stepwise multiple
regression analysis revealed that DM duration and MBPS were independent
predictors associated with increased microalbuminuria. Conclusion: higher
values of MBPS are associated with increased microalbuminuria in
normotensive type 2 diabetic patients.
Keywords: Morning blood pressure surge; Microalbuminuria;
Normotensive; Type 2 diabetes.
without advancing to abnormal values appear to be related to more accelerated cardiovascular complications in patients with type 2 diabetes mellitus (T2DM). Morning blood pressure surge (MBPS) has been increasingly reported to provide a significant predictor of cardiovascular disease (CVD) risk. We aim to study the whether MBPS is associated with increased levels of microalbuminuria in normotensive T2DM patients. Methods: Normotensive T2DM patients were subjected to ambulatory blood pressure monitoring (ABPM) and urine spot sampling to assess urine albumin creatinine ratio (UACR). Results: $32 \%$ of patients had microalbuminuria with a highly significant moderate positive correlation between UACR with DM duration, MBPS and average 2-hours systolic blood pressure $(\mathrm{SBP})$ with $(\mathrm{r}=0.342) \mathrm{p}$-value $<0.01$, $(\mathrm{r}=0.361) \mathrm{p}$-value $<$ regression analysis revealed that DM duration and MBPS were independent predictors associated with increased microalbuminuria. Conclusion: higher values of MBPS are associated with increased microalbuminuria in Keywords: Morning blood pressure surge; Microalbuminuria; Normotensive; Type 2 diabetes

\section{INTRODUCTION}

B lood pressure (BP) decreases in the night [1] and then increases in the morning as the subject awakes and starts daily activities [2]. Unfavorable outcomes were related to changes that happen beyond physiological range. Morning increase and night-time decrease in BP [3-5] were both reported to be associated with cardiovascular morbidity and mortality in both general and diabetic populations [6, 7]. MBPS has been increasingly reported to provide a significant predictor of CVD risk. [8].

The slight fluctuations in BP regulation even without advancing to abnormal values appear to be related to more accelerated cardiovascular complications in patients with T2DM [9]. A few studies with contrasting findings have assessed the relation between MBPS and CV risk in T2DM [10,
11]. So, a better interpretation of the linking between MBPS and CVD is in need to understand if MBPS accelerates vascular damage and leads to more CV events [12].

\section{METHODS}

This was a cross-sectional study conducted in Cardiology Department, Faculty of Medicine, Zagazig University, on adult normotensive diabetic patients attending outpatient clinics of cardiology and diabetes in Zagazig university hospitals in the period between February 2018 and November 2018. Diagnosis of type 2 DM was done according to diagnostic criteria of "American diabetes association (ADA): a fasting plasma glucose level of $126 \mathrm{mg} / \mathrm{dL}$ or higher, a 2-hour plasma glucose level of $200 \mathrm{mg} / \mathrm{dL}$ or higher during a 75 -g oral glucose tolerance test (OGTT) and a random plasma glucose of $200 \mathrm{mg} / \mathrm{dL}$ or higher" [13]. The patients were normotensive 
according to the definition by "European society of cardiology and European society of hypertension (ESC/ESH) guidelines: office SBP $<140 \mathrm{mmHg}$ and DBP $<90 \mathrm{mmHg}$, ABPM mean day SBP $<135 \mathrm{mmHg}$ and DBP $<85 \mathrm{mmHg}$, ABPM mean night SBP < 120 $\mathrm{mmHg}$ and DBP $<70 \mathrm{mmHg}$ and ABPM mean 24 hours SBP $<130 \mathrm{mmHg}$ and DBP < $80 \mathrm{mmHg}$ " [14]. Patients with one or more of the following criteria were excluded from the study: age > 65 years, ischemic heart disease, renal failure, current or history of antihypertensive medications, acute or chronic infectious disease, congestive heart failure, arrhythmias (including atrial fibrillation), valvular heart disease, pregnancy or lactation and prior or current cancer. All patients received full explanation of the study, and each patient delivered a written consent. The study was approved by the medical research and ethics committee of Faculty of Medicine, Zagazig University. The work has been carried out in accordance with The Code of Ethics of the World Medical Association (Declaration of Helsinki) for studies involving humans.

All patients were subjected to complete history taking, General and local examination: with emphasis on:wight and height for calculating body mass index (BMI) and body surface area (BSA), office BP measuremen, examination of pulse, neck veins and lower limbs, local examination of the chest and hear and Electrocardiography (ECG).

After confirming a normal office BP, all patients underwent ABPM using Riester $\mathrm{Ri}$-cardio ambulatory blood pressure monitor validated according to the protocol of ESH. The measurement intervals were 30 minutes during day and night. Patients with more than two hours of missing recordings had another 24 hours ABPM or were excluded from the study. Daytime and sleep-time were defined by diaries filled out by the subject during the test. Mean SBP and DBP of 24 hours, daytime and sleep-time were calculated for each subject. MBPS was defined using the sleep-trough method i.e. the difference between mean SBP in the first two hours of awakening and the mean of three readings centered on the maximum asleep SBP dip [15].

UACR was used for assessment of microalbuminuria. A morning sample was collected from the subject in three different visits, and the results were interpreted according to ADA classification of microalbuminuria based on UACR where two out of three samples with UACR more than $30 \mathrm{mcg} / \mathrm{mg}$ was considered positive for microalbuminuria [16].

\section{Statistical analysis}

Analysis of the collected data was performed using statistical package for the social sciences (SPSS) version 23. The correlation between microalbuminuria and other numerical variables was assessed by Spearman's rank correlation. Stepwise multiple regression analysis was performed to detect the associated independent variables for increase in microalbuminuria. All tests of significance were two-tailed and a p-value < 0.05 was considered statistically significant, a p-value $<0.001$ was considered highly statistically significant and a $p$-value $\geq 0.05$ was considered non-statistically significant.

\section{RESULTS}

Out of 122 patients who underwent ABPM, 7 patients were excluded from the study due to incomplete ABPM readings, 9 patients were excluded due to confirmation of masked hypertension, and 18 patients neglected the follow up visits for urinary sampling. We enrolled the remaining 90 patients into the study.

The mean age of the study group was $(49 \pm 6)$ years. The group had 47 males $(52.2 \%)$ and 43 females $(47.8 \%)$. Further descriptive analysis of the demographic data and ABPM parameters is demonstrated in (tables 1 and 2) respectively.

Regarding categorical variables, there was no significant difference between subjects with and without microalbuminuria regarding gender, smoking and dipping status with p-values (0.530), (0.634) and (0.871) respectively.

Spearman's rank correlation showed a highly statistically significant moderate positive correlation between UACR with DM duration, MBPS and average 2-hours SBP 
with $(\mathrm{r}=0.342) \mathrm{p}$-value $<0.01$, $(\mathrm{r}=0.361) \mathrm{p}$ value $<0.01$ and $(r=0.324)$ p-value $<0.01$ respectively (fig. 1) (table 3).

Stepwise multiple regression analysis generated two models explaining the variance in UACR. The first model showed that DM duration predicts the variance in UACR with $\mathrm{R}=(0.394), \mathrm{R}$ square $=(0.155)$ and adjusted $\mathrm{R}$ square $=(0.145)$ with standardized coefficient $($ Beta $)=(0.394)$ p-value $<0.001$. The second model showed that DM duration combined with MBPS predict the variance in UACR with $\mathrm{R}=(0.457), \mathrm{R}$ square $=(0.209)$ and adjusted $\mathrm{R}$ square $=(0.190)$ with standardized coefficient $($ Beta $)=(0.233) \mathrm{p}$ value 0.017 (table 4 ).

Variables excluded by the stepwise process were age (p-value 0.498), BMI (pvalue 0.575 ), office SBP (p-value 0.299), office DBP (p-value 0.213), HbA1c (p-value 0.623 ), serum creatinine ( $p$-value 0.467), average day SBP (p-value 0.209), average day DBP (p-value 0.872), average asleep SBP (pvalue 0.348), average asleep DBP (p-value 0.267 ), average 24-hour SBP (p-value 0.748), average 24-hour DBP (p-value 0.235), average 2-hour awakening SBP (p-value 0.444 ) and maximum night dip SBP (p-value $0.659)$.

Table 1. Descriptive analysis for patient characteristics and relevant laboratory tests:

\begin{tabular}{|l|l|l|}
\hline \multicolumn{2}{|l|}{ Variable } & Description \\
\hline \multirow{2}{*}{ Age } & Males (\%) & $49.07 \pm 6.087$ years \\
\cline { 2 - 3 } & Females (\%) & $47(52.2 \%)$ \\
\hline BMI & $43(47.8 \%)$ \\
\hline BSA & Smokers (\%) & $27(23-33.2) \mathrm{kg} / \mathrm{m}^{2}$ \\
\hline \multirow{2}{*}{ Smoking } & Non-smokers (\%) & $1.83 \pm 0.17 \mathrm{~m}^{2}$ \\
\hline DM duration & $29(32.2 \%)$ \\
\hline Office SBP & $61(67.8)$ \\
\hline Office DBP & $3(1-5)$ years \\
\hline HbA1c & $116.39 \pm 7.826 \mathrm{mmHg}$ \\
\hline Serum creatinine & $73.28 \pm 6.323 \mathrm{mmHg}$ \\
\hline UACR & $7.1(6.6-7.9) \%$ \\
\hline
\end{tabular}

BMI; body mass index, BSA; body surface area, DM; diabetes mellitus, SBP; systolic blood pressure, DBP; diastolic blood pressure, UACR; urine albumin creatinine ratio.

Table 2. Descriptive analysis for ABPM and MBPS data:

\begin{tabular}{|c|c|c|}
\hline \multicolumn{2}{|l|}{ Variable } & Value (mmHg) \\
\hline \multicolumn{2}{|c|}{ Average day SBP } & $117.74 \pm 8.91$ \\
\hline \multicolumn{2}{|c|}{ Average day DBP } & $77.76 \pm 4.39$ \\
\hline \multicolumn{2}{|c|}{ Average asleep SBP } & $106.58 \pm 7.48$ \\
\hline \multicolumn{2}{|c|}{ Average asleep DBP } & $63.89 \pm 3.5$ \\
\hline \multicolumn{2}{|c|}{ Average 24h SBP } & $114.00 \pm 8.52$ \\
\hline \multicolumn{2}{|c|}{ Average $24 \mathrm{~h}$ DBP } & $71.77 \pm 4.19$ \\
\hline \multicolumn{2}{|c|}{ 2h-awakening SBP } & $128.63 \pm 12.3$ \\
\hline \multicolumn{2}{|c|}{ Maximum night dip SBP } & $101.61 \pm 4.39$ \\
\hline \multirow{4}{*}{ Dipping status } & Normal (\%) & $43(47.8 \%)$ \\
\hline & Extreme (\%) & $7(7.8 \%)$ \\
\hline & Reduced (\%) & $30(33.3 \%)$ \\
\hline & Non-dipping (\%) & $10(11.1 \%)$ \\
\hline \multicolumn{2}{|l|}{ MBPS } & $27.47 \pm 11.097$ \\
\hline
\end{tabular}

SBP; systolic blood pressure, DBP; diastolic blood pressure, MBPS; morning blood pressure surge. 
Table 3. Spearman's correlation of UACR with DM duration, MBPS and average 2 -hours awakening SBP:

\begin{tabular}{|c|c|c|c|c|}
\hline \multicolumn{2}{|l|}{ Spearman's correlation } & DM duration & MBPS & $\begin{array}{c}\text { Average } \\
\text { awakening } \\
\text { SBP }\end{array}$ \\
\hline \multirow{2}{*}{ UACR } & $\begin{array}{c}\text { Correlation } \\
\text { coefficient }\end{array}$ & 0.342 & 0.361 & 0.324 \\
\cline { 2 - 5 } & p-value & $0.001(\mathrm{~S})$ & $<0.001(\mathrm{HS})$ & $0.002(\mathrm{~S})$ \\
\hline
\end{tabular}

DM; diabetes mellitus, MBPS; morning blood pressure surge, UACR; urine albumin creatinine ratio, SBP; systolic blood pressure.

Table 4. Stepwise multiple regression models for UACR predictors in the study group:

\begin{tabular}{|c|c|c|c|c|}
\hline \multicolumn{5}{|c|}{ Regression model for UACR } \\
\hline & $\mathbf{R}$ & R Square & $\begin{array}{c}\text { Adjusted R } \\
\text { Square }\end{array}$ & p- value \\
\hline DM duration & 0.394 & 0.155 & 0.145 & $<0.001(\mathrm{HS})$ \\
\hline DM duration \& MBPS & 0.457 & 0.209 & 0.190 & $0.017(\mathrm{~S})$ \\
\hline
\end{tabular}

UACR; urine albumin creatinine ratio, DM; diabetes mellitus, MBPS; morning blood pressure surge.

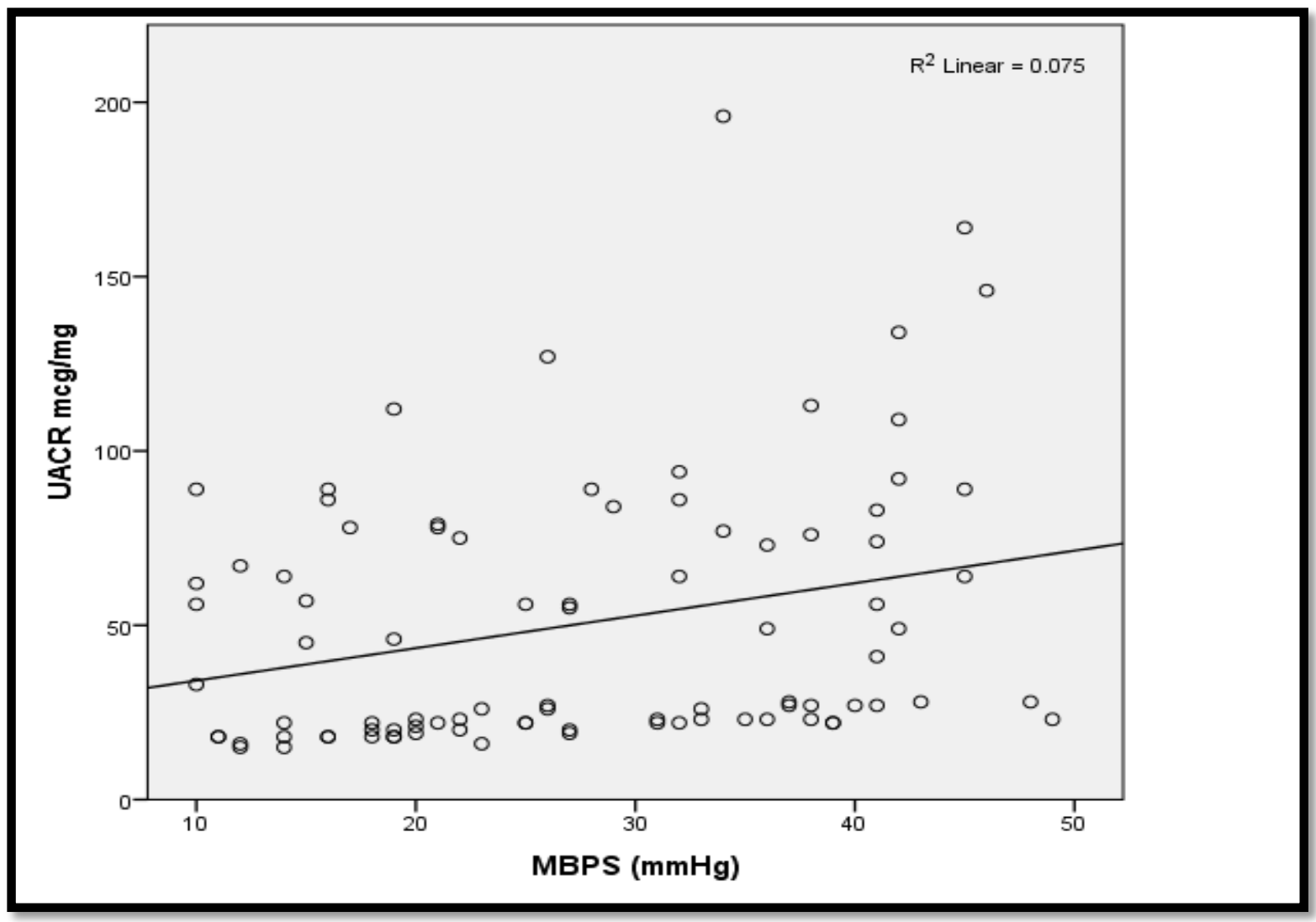

Figure 1. Scatterplot showing intermediate positive correlation between UACR and MBPS: 


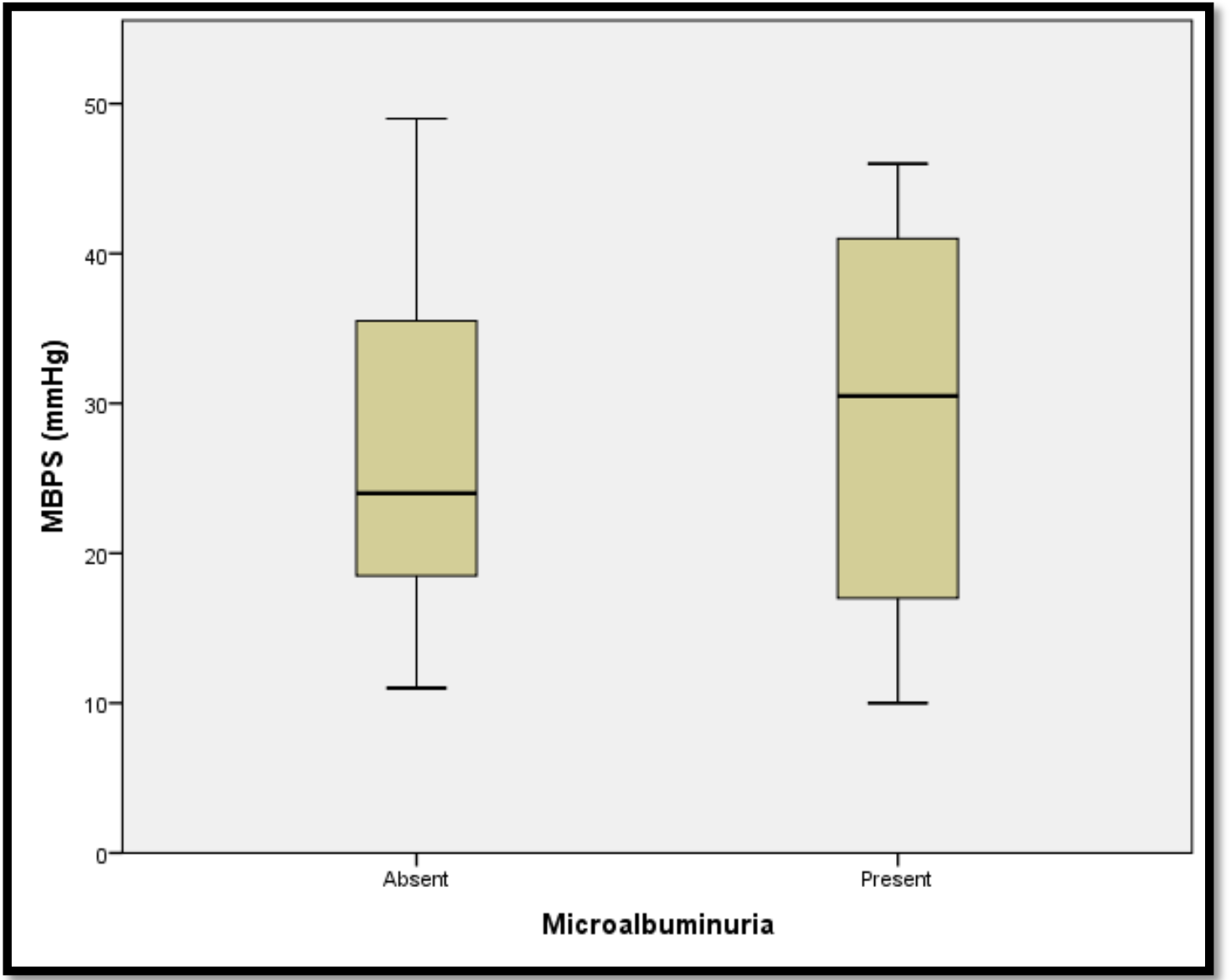

Figure 2. Box plot showing the significant difference in MBPS between subjects without (left) and with (right) microalbuminuria, being significantly higher in subjects with microalbuminuria:

\section{DISCUSSION}

Blood pressure rhythm along 24-hour has been extensively studied since the introduction of ABPM in the investigation and management of hypertension. The normal pattern of BP is described by a morning rise and a night-time fall $[17,18]$.

Increased morning rise and night-time fall were reported to be associated with cardiovascular morbidity and mortality in both general $[3,5]$ and diabetic subjects $[6$, 7]. Even slight alterations in BP pattern seem to be a risk factor for microvascular injury in T2DM [9].

So, our study aimed to find the association between MBPS and microalbuminuria in normotensive T2DM patients.

In this study, there was highly significant positive correlation between MBPS and degree of microalbuminura (expressed as UACR). In a 6.5 years follow up of 377 normotensive patients with T2DM and normal urinary albumin excretion to conclude the impact of MBPS on UACR, it was found that microalbuminuria developed in 102 patients. Consequently, it was concluded that an increased MBPS increases the risk for development of microalbuminuria in adults with normal BP and T2DM [19].

Also, Kramer et al. [20] studied BP rhythm associated with accelerated microvascular injury in T2DM and found that the urinary albumin was significantly correlated to increments in afternoon SBP and DBP. In agreement with our study, it was observed that urinary albumin excretion rate was more correlated with mean SBP than with dipping status in 270 T2DM patients [21].

Non-dipping of nocturnal BP is very often seen in the context chronic kidney disease [22] and this non-dipping status may precede the development of microalbuminuria [23]. In line with our study, Marfella et al. 
[24] demonstrated that higher levels of MBPS were present in patients with microalbuminuria in a cross sectional study. The mechanism of such process was suggested to be a higher intra-glomerular pressure surge in the context of a higher MBPS in the presence of disrupted autoregulation of the afferent arterioles brought by the diabetic process [25].

On the other hand, it was found that MBPS was not associated with urinary albumin in recently diagnosed type $2 \mathrm{DM}$ patients [26]. This may be related to the general difficulty in determining an accurate onset for T2DM. Therefore, some patients may present with advanced microalbuminuria with confirmed diabetic nephropathy on kidney biopsy before or recently after T2DM is diagnosed [27].

Actually, the wide variety of results among researchers may be due to the different methods and thresholds for defining MBPS [28]. In addition, ethnicity [29], sex, age, BMI $[30,31]$, hypertension [31], use of $\beta$-blockers $[31,32]$, a history of cardiovascular disease [30, 33], duration of diabetes mellitus [30], renal dysfunction, a sedentary lifestyle [33], and socioeconomic position [34] all are factors can affect the measured parameters.

This proposes that the relationship between MBPS and CVD is more complex than simply using a single threshold and is unsurprising given that analysis of subject's continuous predictors on scale is more accurate and is less liable to error than dealing with a certain threshold as a categorical entity [28].

\section{CONCLUSION}

Higher levels of MBPS are associated with increased incidence of microalbuminuria in normotensive type 2 diabetic patients independent of office and average ambulatory $\mathrm{BP}$ values and dipping status in our study.

\section{Declaration of interest}

The authors report no conflicts of interest. The authors alone are responsible for the content and writing of the paper.

Funding information None declared

\section{REFERENCES}

1. Muller JE, Stone PH, Turi ZG, Rutherford JD, Czeisler CA, Parker C, et al. Circadian variation in the frequency of onset of acute myocardial infarction. New England journal of medicine. 1985;313(21):1315-1322.

2. Mancia G, Ferrari A, Gregorini L, Parati G, Pomidossi G, Bertinieri G, et al. Blood pressure and heart rate variabilities in normotensive and hypertensive human beings. Circulation research. 1983;53(1):96-104.

3. Izzedine $\mathrm{H}$, Launay-Vacher $\mathrm{V}$, Deray $\mathrm{G}$. Abnormal blood pressure circadian rhythm: a target organ damage? International journal of cardiology. 2006;107(3):343-349.

4. Jones H, Atkinson G, Leary A, George K, Murphy M, Waterhouse J. Reactivity of ambulatory blood pressure to physical activity varies with time of day. Hypertension. 2006;47(4):778-784.

5. Ohkubo T, Hozawa A, Yamaguchi J, Kikuya M, Ohmori K, Michimata M, et al. Prognostic significance of the nocturnal decline in blood pressure in individuals with and without high 24-h blood pressure: the Ohasama study. J Hypertens. 2002;20(11):2183-2189.

6. Pecis M, Azevedo MJ, Moraes RS, Ferlin EL, Gross JL. Autonomic dysfunction and urinary albumin excretion rate are associated with an abnormal blood pressure pattern in normotensive normoalbuminuric type 1 diabetic patients. Diabetes care. 2000;23(7):989-993.

7. Sturrock N, George E, Pound N, Stevenson J, Peck G, Sowter H. Non-dipping circadian blood pressure and renal impairment are associated with increased mortality in diabetes mellitus. Diabetic medicine. 2000;17(5):360364.

8. White WB. The risk of waking-up: impact of the morning surge in blood pressure. Hypertension. 2010;55(4):835-837.

9. Kramer CK, Leitão CB, Canani LH, Ricardo ED, Pinto LC, Valiatti FB, et al. Late afternoon blood pressure increase is associated with diabetic retinopathy in normotensive type 2 diabetes mellitus patients. Diabetes research and clinical practice. 2009;84(1):e12-e14.

10.Eguchi K, Ishikawa J, Hoshide S, Pickering TG, Schwartz JE, Shimada K, et al. Night time blood pressure variability is a strong predictor for cardiovascular events in patients with type 2 diabetes. American journal of hypertension. 2009;22(1):46-51.

11.Hermida RC, Ayala DE, Mojón A, Fernández JR. Sleep-time blood pressure as a therapeutic target for cardiovascular risk reduction in type 2 diabetes. American journal of hypertension. 2012;25(3):325-334. 
12. Yoda $K$, Inaba $M$, Hamamoto $K$, Yoda $M$, Tsuda A, Mori K, et al. Association between glycemic control and morning blood pressure surge with vascular endothelial dysfunction in type 2 diabetic patients. Diabetes care. 2014;37(3):644-650.

13.American Diabetes Association. Standards of medical care for patients with diabetes mellitus. Diabetes care. 2003;26(suppl 1):s33s50.

14.Williams B, Mancia G, Spiering W, Agabiti Rosei E, Azizi M, Burnier M, et al. 2018 ESC/ESH Guidelines for the management of arterial hypertension: The Task Force for the management of arterial hypertension of the European Society of Cardiology and the European Society of Hypertension: The Task Force for the management of arterial hypertension of the European Society of Cardiology and the European Society of Hypertension. J Hypertens. 2018;36(10):19532041.

15.Kario K. Caution for winter morning surge in blood pressure: a possible link with cardiovascular risk in the elderly. Hypertension. 2006;47(2):139-140.

16. American Diabetes Association. 10. Microvascular complications and foot care: standards of medical care in diabetes-2018. Diabetes care. 2018;41(Supplement 1):S105S118.

17.Kario K. New Insight of Morning Blood Pressure Surge Into the Triggers of Cardiovascular Disease-Synergistic Resonance of Blood Pressure Variability. American journal of hypertension. 2015;29(1):14-16.

18.Muller JE. Circadian variation in cardiovascular events. American journal of hypertension. 1999;12(S2):35S-42S.

19.Barbieri M, Rizzo MR, Fava I, Sardu C, Angelico N, Paolisso P, et al. Awaking blood pressure surge and progression to microalbuminuria in type 2 normotensive diabetic patients. Journal of diabetes research. 2016;2016.

20.Kramer CK, Leitão CB, Canani LH, Gross JL. Afternoon blood pressure increase: a blood pressure pattern associated with microvascular complications in type 2 diabetes mellitus. American journal of hypertension. 2011;24(1):64-69.

21.Leitão C, Canani L, Kramer C, Moehlecke M, Pinto L, Ricardo E, et al. Blood pressure means rather than nocturnal dipping pattern are related to complications in Type 2 diabetic patients. Diabetic medicine. 2008;25(3):308313.

22.Kimura G. Kidney and circadian blood pressure rhythm. Hypertension. 2008;51(4):827.

23.Lurbe E, Redon J, Kesani A, Pascual JM, Tacons $\mathrm{J}$, Alvarez $\mathrm{V}$, et al. Increase in nocturnal blood pressure and progression to microalbuminuria in type 1 diabetes. New England journal of medicine. 2002;347(11):797-805.

24.Marfella R, Esposito K, Giugliano D. Increase in nocturnal blood pressure and progression to microalbuminuria in diabetes. New England journal of medicine. 2003;348:260-264.

25.Kario K. Morning surge in blood pressure and cardiovascular risk: evidence and perspectives. Hypertension. 2010;56(5):765-773.

26.Lyhne JM, Laugesen E, Høyem P, Cichosz S, Christiansen JS, Knudsen ST, et al. Morning blood pressure surge and target organ damage in newly diagnosed type 2 diabetic patients: a cross sectional study. BMC endocrine disorders. 2015;15(1):77.

27.Umanath K, Lewis JB. Update on diabetic nephropathy: core curriculum 2018. American Journal of Kidney Diseases. 2018;71(6):884895.

28. Sheppard JP, Hodgkinson J, Riley R, Martin $\mathrm{U}$, Bayliss S, McManus RJ. Prognostic significance of the morning blood pressure surge in clinical practice: a systematic review. American journal of hypertension. 2014;28(1):30-41.

29. White WB. Diagnostic evaluation: ambulatory blood pressure monitoring in clinical hypertension management. Journal of the American Society of Hypertension. 2014;8(12):939-941.

30.De la Sierra A, Redon J, Banegas J, Segura J, Parati G, Gorostidi M, et al. Spanish Society of Hypertension Ambulatory Blood Pressure Monitoring Registry I. Prevalence and factors associated with circadian blood pressure patterns in hypertensive patients. Hypertension. 2009;53:466-472.

31. Schutte R, Thijs L, Liu Y-P, Asayama K, Jin Y, Odili A, et al. Within-subject blood pressure level — not variability-predicts fatal and nonfatal outcomes in a general population. Hypertension. 2012:HYPERTENSIONAHA. 112.202143.

32.Rothwell PM, Howard SC, Dolan E, O'Brien E, Dobson JE, Dahlöf B, et al. Effects of $\beta$ blockers and calcium-channel blockers on within-individual variability in blood pressure 
and risk of stroke. The Lancet Neurology. 2010;9(5):469-480.

33. Muntner P, Shimbo D, Tonelli M, Reynolds K, Arnett DK, Oparil S. The relationship between visit-to-visit variability in systolic blood pressure and all-cause mortality in the general population: findings from NHANES III, 1988 to $1994 . \quad$ Hypertension. 2011:HYPERTENSIONAHA. 110.162255.
34.Hickson DA, Roux AVD, Wyatt SB, Gebreab SY, Ogedegbe G, Sarpong DF, et al. Socioeconomic position is positively associated with blood pressure dipping among African-American adults: the Jackson Heart Study. American journal of hypertension. 2011;24(9):1015-1021.

To cite this article: Mahfouz RA, Shehata IE, Ibrahim IM , Abdel-Kareem AO*. Assessment of B-cell Activating Factor (BAFF) in children with Acute Lymphoblastic Leukemia.Zumj May. 2020(26) No.3,442449.DOI: 10.21608 /zumj.2019.17149.1529. 